\title{
Reading blood glucose from subcutaneous electric current by means of a regularization in variable Reproducing Kernel Hilbert Spaces
}

\author{
Valeriya Naumova, Sergei V. Pereverzyev and Sivananthan Sampath
}

\begin{abstract}
In this paper we propose an adaptive kernel regularization algorithm for blood glucose reading from subcutaneous electric current. We illustrate the proposed algorithm with clinical data and quantify its clinical accuracy by means of the Clarke error grid analysis (EGA) and by the number of detected hypoglycemic events. We show that the proposed algorithm provides more accurate blood glucose reading than a commercially available system.
\end{abstract}

\section{INTRODUCTION}

Diabetes mellitus is a common and serious disease in which blood glucose levels abnormally fluctuate. Moreover, diabetes is a prime risk factor for heart disease, kidney failure, eye problems, brain damage, coma and diabetic hyperosmolar syndrome. It has been shown in the Diabetes Controls and Complication Trial (DCCT) [1] that there are several advantages if diabetes patients control blood glucose levels at close to normal values. Moreover, bolus calculators, which are extensively used in diabetes management, also rely on the knowledge of current blood glucose level [2]. Hence, in order to control blood glucose evolution within the normal range, it is important to know the current blood glucose level.

Minimal-invasive continuous glucose monitoring (CGM) systems are developed to provide estimated blood glucose values almost in real-time in replacement of painful finger sticks measurements. Some of the approved and commercially available CGM sensors are Medtronic's Minimed device, DexCom and Abbott Freestyle Navigator. The needle based electrochemical sensors, for example Abbott Freestyle Navigator, measure electrical signal (ADC counts) in the interstitial fluid (ISF) and return estimated current blood glucose concentration $(\mathrm{mg} / \mathrm{dL})$ exploiting some internal calibration procedure.

At the same time, it is known [3] that the equilibration between blood and ISF glucose is not instantaneous. Therefore, sometimes CGM systems provide a distorted estimated blood glucose level.

In [3] it has been shown how such a distortion can be compensated through the use of a mathematical model of blood-interstitium kinetics. Within this model a blood glucose concentration can be recovered from ISF glucose level provided that the latter one was accurately determined

V. Naumova, S. V. Pereverzyev and S. Sampath, Johann Radon Institute for Computational and Applied Mathematics (RICAM), Austrian Academy of Sciences, Altenbergerstrasse 69, A-4040 Linz, Austria valeriya.naumova@oeaw.ac.at, sergei.pereverzyev@oeaw.ac.at,

sivananthan.sampath@oeaw.ac.at from subcutaneous electric current measurements by means of a calibration procedure.

The results of [3] show that in this approach the role of calibration is really crucial. In particular, these results suggest that further improvements of blood glucose reconstruction require more sophisticated procedures than the standard calibration by which ISF glucose is determined in CGM systems, such as Abbott Freestyle Navigator.

In this paper we propose to develop such a procedure on the base of regularization in variable Reproducing Kernel Hilbert Spaces (RKHSs).

The problem can be stated as follows: we are given a sample set $\mathbf{z}=\left\{\left(x_{i}, y_{i}\right)\right\}_{i=1}^{n}$, where $x_{i}$ denotes electric current in ISF (ADC counts), and $y_{i}$ denotes blood glucose concentration $(\mathrm{mg} / \mathrm{dL})$ measured at the same time. The goal is to find a function $f$ that minimizes the given data error, for example,

$$
f:=\arg \min _{g \in \mathcal{H}} \frac{1}{|\mathbf{z}|} \sum_{i=1}^{|\mathbf{z}|}\left(y_{i}-g\left(x_{i}\right)\right)^{2},
$$

where $|\mathbf{z}|$ is the cardinality of the set $\mathbf{z}$, i.e. $|\mathbf{z}|=n$, and $\mathcal{H}$ is some class of functions. However, in practice we do not know the class $\mathcal{H}$, and also the given measurements are not exact.

Most of the calibration procedures assume that the relation between blood and ISF glucose levels is linear. In such a case, $\mathcal{H}$ can be chosen as the space of linear polynomials [4]. But we do know that such a model is too simplistic [3].

At the same time, if we choose $\mathcal{H}$ to be too large then the function $f$ in (1) will interpolate the given noisy data, and we end up in overfitting. Therefore, one has to trade-off between the data fitting and the complexity of the data fitter. This problem can be solved by using the following Tikhonovtype functional with $\mathcal{H}$ to be an RKHS $\mathcal{H}_{K}$, defined by a positive definite function $K$,

$$
T_{K, \lambda, \mathbf{z}}(f)=\frac{1}{|\mathbf{z}|} \sum_{i=1}^{|\mathbf{z}|}\left(y_{i}-f\left(x_{i}\right)\right)^{2}+\lambda\|f\|_{K}^{2},
$$

where $\|f\|_{K}^{2}$ is the norm in $\mathcal{H}_{K}$, and $\lambda$ is a regularization parameter [5]. Then the blood glucose estimator $f=f_{K, \mathbf{z}}^{\lambda}$ can be given by

$$
f_{K, \mathbf{z}}^{\lambda}:=\arg \min _{f \in \mathcal{H}_{K}} T_{K, \lambda, \mathbf{z}}(f) .
$$

Use of the Tikhonov regularization scheme (2), (3) raises two issues to be concerned about. One of them is how to choose a regularization parameter $\lambda$ in (3). For a fixed kernel $K$, the 
choice of the regularization parameter $\lambda$ has been extensively studied [6], [7], [8].

Another issue is how to choose the regularization space $\mathcal{H}_{K}$ or, that is the same thing, as the choice of the kernel $K$ generating this space. Despite of its importance, this issue has begun to be discussed only recently (see for example, [8], [9], [10], [11]).

In the present paper we partly use the theoretical results of [11], where these two issues have been addressed in the context of the function reconstruction from a given data set, in particular, for blood glucose reading. However, here we concentrate our attention more on the problem of improvement of the blood glucose estimation from subcutaneous electric current. The main novelty of this paper is a new criterion for the kernel choice, which is oriented towards more accurate blood glucose reading in dangerous glycemic ranges (see, for example, Fig. 1). In experiments with clinical data a blood glucose reader based on this criterion outperforms a commercially available CGM-device (Abbott Freestyle Navigator) that uses the same electric current measurements (see Tables I and III). A surprising by-product is that the proposed criterion leads to a patient independent kernel choice, at least for available clinical data.

The paper is organized as follows. In section 2, we discuss a theoretical background for regularization in variable RKHSs. In section 3, we adjust the approach of [11] to the blood glucose reading by introducing a new form of the minimization functional. Further, we illustrate the proposed algorithm with clinical data and discuss its clinical accuracy.

\section{REgUlaRiZATION IN VARIABLE REPRODUCING KeRnEl Hilbert SPACES}

Let $X$ be a subset of $\mathbb{R}$. A function $K: X \times X \rightarrow \mathbb{R}$ is called a kernel if for all finite subsets $\mathbf{x}:=\left\{x_{j}: j=\right.$ $1, \ldots, n, n \in \mathbb{N}\} \subset X$ the matrix $\mathbb{K}[\mathbf{x}]:=\left[K\left(x_{i}, x_{j}\right)\right]_{i, j=1}^{n}$ is symmetric and positive definite.

A Hilbert space $\mathcal{H}$ of real valued functions on $X$ with an inner product $\langle\cdot, \cdot\rangle$ is called a Reproducing Kernel Hilbert Space if for any $x \in X$ the functional $\mathcal{F}_{x}$ defined as

$$
\mathcal{F}_{x}(f)=f(x)
$$

is bounded. As a consequence of the Riesz representation theorem, there exists a unique kernel $K(x, u)$ on $X$, called a reproducing kernel of $\mathcal{H}$, which has the following reproducing property

$$
f(x)=\langle f, K(x, \cdot)\rangle, \quad \forall f \in \mathcal{H} .
$$

Conversely, if $K$ is a kernel on $X$ then there exists a unique RKHS $\mathcal{H}$ such that $K(x, \cdot) \in \mathcal{H}, \forall x \in \mathcal{H}$, and it has the reproducing property (4) [12]. Therefore, there is one-to-one correspondence between the set of kernels and the set of RKHSs. Thus, determining the RKHS $\mathcal{H}=\mathcal{H}_{K}$ is equivalent to determining the corresponding kernel $K$.

For a given data $\mathbf{z}=\left\{\left(x_{i}, y_{i}\right)\right\}_{i=1}^{n} \subset X \times \mathbb{R}$, consider the minimization problem (3). Then from the Representer theorem [5] the function $f_{K, \mathbf{z}}^{\lambda}$ can be explicitly written as

$$
f_{K, \mathbf{z}}^{\lambda}(x)=\sum_{i=1}^{|\mathbf{z}|} c_{i}^{\lambda} K\left(x_{i}, x\right),
$$

where the vector of coefficients $\mathbf{c}=\left(c_{i}^{\lambda}\right)_{i=1}^{|\mathbf{z}|}$ satisfies $\mathbf{c}=(\mathbb{K}[\mathbf{x}]+\lambda|\mathbf{z}| \mathbb{I})^{-1} \mathbf{y}$, here $\mathbb{I}$ is the identity matrix of order $|\mathbf{z}|$ and $\mathbf{y}=\left(y_{i}\right)_{i=1}^{|\mathbf{z}|}$.

Regularization in an RKHS has a number of attractive features, including the effective error bounds and stability analysis relative to perturbations of the data. For further detailed information, please refer to [13], [14], [15].

From equation (5), it is clear that if one provides an appropriate kernel $K$ and a regularization parameter $\lambda$ for a given data $\mathbf{z}$ then one can compute the regularized approximant $f_{K, \mathbf{z}}^{\lambda}$. In the following subsections, we discuss a choice of the regularization parameter $\lambda$ and the kernel $K$.

\section{A. A posteriori regularization parameter choice}

Suppose a kernel $K$ is fixed, then an appropriate choice of the regularization parameter $\lambda$ is important to get a good performance of (3). For example, one can use a data-driven method for choosing the regularization parameter called the quasi-balancing principle [8]. This heuristic principle can be seen as a combination of the balancing-principle [8] and the quasi-optimality criterion [6]. In [8] and [16] it has been shown that the balancing-principle and the quasi-optimality criterion may potentially give an accuracy of optimal order for a given kernel $K$.

To apply the quasi-balancing principle [8] one needs to calculate the approximations $f_{K, \mathbf{z}}^{\lambda}$ given by (5) for $\lambda$ from a finite geometric sequence

$$
\Lambda_{q}^{\nu}=\left\{\lambda_{s}=\lambda_{0} q^{s}, s=0,1,2, \ldots, \nu\right\}, q>1 .
$$

Then one needs to calculate the norms

$$
\begin{aligned}
\sigma_{e m p}^{2}(s) & =\left\|f_{K, \mathbf{z}}^{\lambda_{s}}-f_{K, \mathbf{z}}^{\lambda_{s-1}}\right\|_{\left\{x_{i}\right\}_{i=1}^{|\mathbf{z}|}}^{2} \\
& :=\frac{1}{|\mathbf{z}|} \sum_{i=1}^{|\mathbf{z}|}\left(\sum_{j=1}^{|\mathbf{z}|}\left(c_{j}^{\lambda_{s}}-c_{j}^{\lambda_{s-1}}\right) K\left(x_{i}, x_{j}\right)\right)^{2}, \\
\sigma_{\mathcal{H}_{K}}^{2}(s) & =\left\|f_{K, \mathbf{z}}^{\lambda_{s}}-f_{K, \mathbf{z}}^{\lambda_{s-1}}\right\|_{K}^{2} .
\end{aligned}
$$

and find

$$
\begin{aligned}
\lambda_{e m p} & =\lambda_{h}, h=\arg \min \left\{\sigma_{e m p}^{2}(s), s=1,2, \ldots, \nu\right\}, \\
\lambda_{\mathcal{H}_{K}} & =\lambda_{p}, p=\arg \min \left\{\sigma_{\mathcal{H}_{K}}^{2}(s), s=1,2, \ldots, \nu\right\} .
\end{aligned}
$$

Finally, in accordance with the quasi-balancing principle a value of the regularization parameter $\lambda=\lambda_{+} \in \Lambda_{q}^{\nu}$ is given as

$$
\lambda_{+}=\min \left\{\lambda_{e m p}, \lambda_{\mathcal{H}_{K}}\right\} .
$$

As it can be easily seen, the choice of the regularization parameter is fully governed by the kernel and input data. 


\section{B. Extrapolation-oriented choice of the kernel}

A choice of the kernel depends on the intended use of $f_{K, \mathbf{z}}^{\lambda}$. This choice can make a significant difference in practice. Thus, it is important to have a criterion to choose a suitable kernel for a given problem.

Ideally, the kernel should be 'learned' from the data. The simplest way of doing this is to take a rich parameterized set of kernels and choose the values of the parameters by some prescribed criteria.

In [9] and [17] the authors considered the set $\mathcal{K}$ of linear combinations of some prescribed kernels $\left\{K_{i}\right\}_{i=1}^{l}$ and proposed various criteria how to select the kernel from the set $\mathcal{K}$. However, for some practical applications such a set of admissible kernels may not be rich enough.

In [10] the authors proposed to choose a kernel $K$ by minimizing the value $T_{K, \lambda, \mathbf{z}}\left(f_{K, \mathbf{z}}^{\lambda}\right)$ over a set of admissible kernels $\mathcal{K}$.

In the recent paper [11], it has been demonstrated that the method of [10] may not be suitable for extrapolation problem. Therefore, as an alternative, the kernel adaptive regularization (KAR) algorithm has been proposed in [11]. It is oriented towards extrapolation and based on splitting of available data $\mathbf{z}$. In general form it can be described as follows.

The given data set $\mathbf{z}=\left\{\left(x_{i}, y_{i}\right)\right\}_{i=1}^{|\mathbf{z}|}$ is splitted as $\mathbf{z}=$ $\mathbf{z}_{T} \cup \mathbf{z}_{P}$ such that

$$
\overline{\operatorname{co}\left\{x_{i}:\left(x_{i}, y_{i}\right) \in \mathbf{z}_{T}\right\}} \cap\left\{x_{i}:\left(x_{i}, y_{i}\right) \in \mathbf{z}_{P}\right\}=\emptyset,
$$

where $\overline{c o\left\{x_{i}\right\}}$ is the closed convex hull of data points $\left\{x_{i}\right\}$.

Then for the fixed $\mathbf{z}_{T}$ and corresponding Tikhonov-type regularization functional

$$
T_{K, \lambda, \mathbf{z}_{T}}(f)=\frac{1}{\left|\mathbf{z}_{T}\right|} \sum_{\left(x_{i}, y_{i}\right) \in \mathbf{z}_{T}}\left(y_{i}-f\left(x_{i}\right)\right)^{2}+\lambda\|f\|_{K}^{2},
$$

one considers a rule $\lambda=\lambda(K)$ that for any kernel $K$ on $X$ selects a regularization parameter from some fixed interval $\left[\lambda_{\min }, \lambda_{\max }\right], \lambda_{\min }>0$. Using the set $\mathbf{z}_{T}$, one constructs the regularized approximant

$$
f_{K, \mathbf{z}_{T}}^{\lambda}=\arg \min T_{K, \lambda, \mathbf{z}_{T}}(f), \quad \lambda=\lambda(K),
$$

and measures its extrapolation performance on the set $\mathbf{z}_{P}$ by the value of the functional $P\left(f_{K, \mathbf{z}_{T}}^{\lambda}, \mathbf{z}_{P}\right)$, where

$$
P\left(f, \mathbf{z}_{P}\right)=\frac{1}{\left|\mathbf{z}_{P}\right|} \sum_{\left(x_{i}, y_{i}\right) \in \mathbf{z}_{P}} \rho\left(f\left(x_{i}\right), y_{i}\right),
$$

and $\rho(\cdot, \cdot)$ is a continuous non-negative function of two variables. Then the kernel $K$ is chosen as the minimizer of the following functional

$$
Q_{\mu}(K, \lambda, \mathbf{z})=\mu T_{K, \lambda, \mathbf{z}_{T}}\left(f_{K, \mathbf{z}_{T}}^{\lambda}\right)+(1-\mu)\left(P\left(f_{K, \mathbf{z}_{T}}^{\lambda}, \mathbf{z}_{P}\right)\right)
$$

over the set of admissible kernels $\mathcal{K}$, for example,

$\mathcal{K}=\left\{K(x, u)=(x u)^{\alpha}+\beta e^{-\gamma(x-u)^{2}}, \alpha, \beta, \gamma \in\left[10^{-4}, 3\right]\right\}$.

Note that the parameter $\mu \in[0,1]$ in (12) can be seen as a performance regulator on the sets $\mathbf{z}_{T}$ and $\mathbf{z}_{P}$. Taking $\mu$ closer to zero we put more emphasize on the ability to extrapolate, while for $\mu>\frac{1}{2}$ we are more interested in interpolation.

It has been shown in [11] that the existence of a kernel of choice can be guaranteed for a rather general form of set $\mathcal{K}$.

Theorem 2.1: Let $\mathcal{K}(X)$ be the set of all kernels defined on $X, \Omega$ be a compact metric space, and $G: \Omega \rightarrow \mathcal{K}(X)$ be a continuous map in the sense that for any $x, u \in X$ the function $w \mapsto K_{w}(x, u) \in \mathbb{R}$ is continuous on $\Omega$, where for $w \in \Omega$ the kernel $K_{w} \in \mathcal{K}(X)$ is given as $K_{w}=G(w)$. Define

$$
\mathcal{K}=\mathcal{K}(\Omega, G)=\{K: K=G(w), K \in \mathcal{K}(X), w \in \Omega\}
$$

to be the set of kernels parameterized via $G$ by elements of $\Omega$.

Then for any parameter choice rule $\lambda=\lambda(K)$ there are $K^{0} \in \mathcal{K}(\Omega, G)$ and $\lambda^{0} \in\left[\lambda_{\min }, \lambda_{\max }\right]$ such that

$Q_{\mu}\left(K^{0}, \lambda^{0}, \mathbf{z}\right)=\inf \left\{Q_{\mu}(K, \lambda(K), \mathbf{z}), K \in \mathcal{K}(\Omega, G)\right\}$. The proof of the Theorem can be found in [11].

Since the problem of the blood glucose reading can be seen as a particular example of extrapolation, we use the approach of [11] to choose a kernel from the set (13) and then use it for constructing a blood glucose reader.

\section{READING BLOOD GLUCOSE FROM SUBCUTANEOUS ELECTRIC CURRENT}

The possibility to use the Theorem 2.1 in the context of the blood glucose reading has been already discussed in [11]. In that paper the form of the functional (11) has been inspired by the notion of the risk function $r(\cdot)$, that has been introduced similar to [18]. In terms of this function the functional (11) has been written in [11] as follows

$$
\left.P\left(f, \mathbf{z}_{P}\right)=\frac{1}{\left|\mathbf{z}_{P}\right|} \sum_{\left(x_{i}, y_{i}\right) \in \mathbf{z}_{P}} \mid y_{i}-f\left(x_{i}\right)\right) \mid w\left(y_{i}, f\left(x_{i}\right)\right),
$$

where $w(u, v)=\frac{|r(u)-r(v)|}{r(v)}+1$. In the experiments with clinical data it has turned out that a kernel, that was found as the minimizer of (12), (14) for data $\mathbf{z}$ of one patient, does not allow a clinically acceptable blood glucose reading for another one's. It means that for each patient the kernel choice procedure based on the minimization of the functionals (12), (14) should be repeated. This may be seen as a disadvantage. Here we propose another form of the functional (11) that not only leads to more accurate blood glucose reading, but also, as a by-product, allows a patient independent kernel choice. Define

$$
P\left(f, \mathbf{z}_{P}\right)=\frac{1}{\left|\mathbf{z}_{P}\right|} \sum_{\left(x_{i}, y_{i}\right) \in \mathbf{z}_{P}}\left|y_{i}-f\left(x_{i}\right)\right|_{A, \epsilon},
$$

where the quantity $\left|y_{i}-f\left(x_{i}\right)\right|_{A, \epsilon}$ is aimed at penalizing the overestimation of low glucose levels, as well as the underestimation of high ones. It is set out as follows:

Case-1 if $y_{i}<70(\mathrm{mg} / \mathrm{dL})$ and for sufficiently small $\epsilon$, say $\epsilon=5(\mathrm{mg} / \mathrm{dL})$, we have $y_{i}+\epsilon<f\left(x_{i}\right)$, then we put $\left|y_{i}-f\left(x_{i}\right)\right|_{A, \epsilon}=A$, where $A$ is large enough.

Case-2 if $f\left(x_{i}\right) \leq y_{i}<70(\mathrm{mg} / \mathrm{dL})$ then $\left|y_{i}-f\left(x_{i}\right)\right|_{A, \epsilon}=$ $y_{i}-f\left(x_{i}\right)$. 
Case-3 in the range of euglycemia, when $70 \leq y_{i} \leq 180$ (mg/dL), we put $\left|y_{i}-f\left(x_{i}\right)\right|_{A, \epsilon}=\left|y_{i}-f\left(x_{i}\right)\right|$.

Case-4 if $y_{i}>180(\mathrm{mg} / \mathrm{dL})$ and $y_{i}-\epsilon>f\left(x_{i}\right)$, we put $\left|y_{i}-f\left(x_{i}\right)\right|_{A, \epsilon}=A$.

Case-5 if $180<y_{i} \leq f\left(x_{i}\right)(\mathrm{mg} / \mathrm{dL})$ then $\left|y_{i}-f\left(x_{i}\right)\right|_{A, \epsilon}=$ $f\left(x_{i}\right)-y_{i}$.

Otherwise, we define the penalizing quantity $\left|y_{i}-f\left(x_{i}\right)\right|_{A, \epsilon}$ by linear interpolation between 0 and $A$ to make it continuously depending on $\left(y_{i}-f\left(x_{i}\right)\right)$, since such continuity is necessary for the application of the Theorem 2.1.

Let us summarize the proposed approach. To select a kernel $K^{0}$ from a given class $\mathcal{K}=\mathcal{K}(\Omega, G)$ one needs a training data set $\mathbf{z}=\left\{\left(x_{i}, y_{i}\right)\right\}_{i=1}^{n}$ consisting of values of the subcutaneous electric current $x_{i}$ and values of the blood glucose concentration $y_{i}$ measured at the same moments of time. This training set $\mathbf{z}$ is splitted into two parts $\mathbf{z}_{T}$ and $\mathbf{z}_{P}$. The first one is used for constructing regularized blood glucose readers $f_{K, \mathbf{z}_{T}}^{\lambda}$ as the minimizers of (10) for $K \in \mathcal{K}(\Omega, G)$ and $\lambda=\lambda(K)$. The performance of these readers is measured on the second data set $\mathbf{z}_{P}$ by the values of the functional (15). In accordance with the Theorem 2.1, there exist a kernel $K^{0} \in \mathcal{K}(\Omega, G)$ and $\lambda=\lambda^{0}$ that optimize the weighted performance in the sense of (12), (15). Then $K^{0}$ is the kernel of our choice, and for any current value $x$ the regularized blood glucose reading is given as $f_{K^{0}, \mathbf{z}}^{\lambda\left(K^{0}\right)}(x)$. In our experiments the role of $\mathcal{K}(\Omega, G)$ is played by the set (13), and for each $K \in \mathcal{K}(\Omega, G)$ the value $\lambda=\lambda(K)$ is chosen in accordance with the quasi-balancing principle (6)-(9).

To illustrate this approach we use data sets of nine diabetic subjects studied in the Montpellier University Hospital Center (CHU, France) and in the Department of Clinical and Experimental Medicine at the University of Padova (UNIPD, Italy) within the framework of EU-project "DIAdvisor" [19].

For each subject blood glucose concentration and subcutaneous electric current were measured in parallel for 3 days in hospital conditions. The blood glucose concentration was measured at least 30 times per day by the HemoCue glucose meter. Blood samples were collected every hour during a day, every 2 hours during a night, every 15 minutes after meals for 2 hours. Specific sampling schedule was adopted after breakfast: 30 minutes before mealtime, 10, 20, 30, 60, 90, 120, 150, 180, 240, 300 minutes after. Subcutaneous electric current was measured every 1 minute by the same needle based sensor that is used in the Abbott Freestyle Navigator.

For each subject the values of the blood glucose concentration and subcutaneous electric current measured at the same time during the first day were used as a training data $\mathbf{z}=\left\{\left(x_{i}, y_{i}\right)\right\}_{i=1}^{30}$. Here $x_{i} \in[1,1024]$ are the current values (ADC counts), while $y_{i} \in[0,450]$ are corresponding values of the blood glucose concentration $(\mathrm{mg} / \mathrm{dL})$.

The training set $\mathbf{z}$ corresponding to the subject CHU102 was used for choosing the kernel $K^{0}$ from the set (13). For this purpose, the set $\mathbf{z}$ was sorted in increasing order of $x_{i}$ and then splitted into two parts, namely $\mathbf{z}_{P}=$ $\left\{\left(x_{i}, y_{i}\right)\right\},\left|\mathbf{z}_{P}\right|=4$, is formed by two first and two last

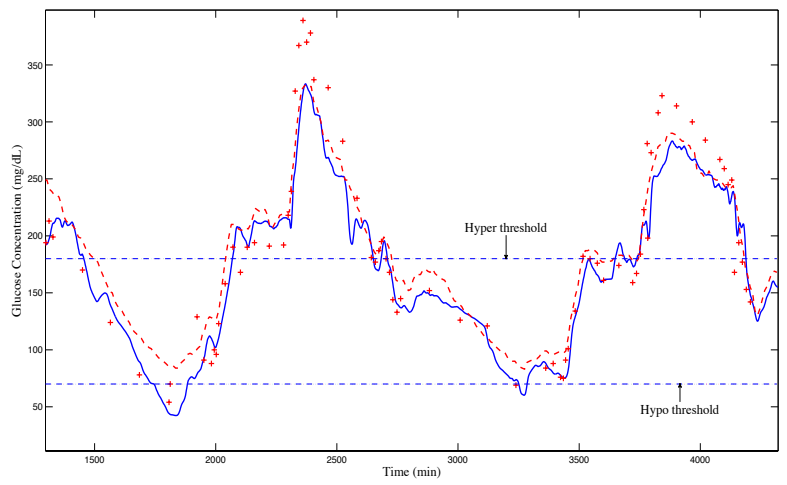

Fig. 1. A graph plot of blood glucose reading from subcutaneous electric current for Subject ID - UNIPD203: The solid line is formed by blood glucose estimations produced by the regularized blood glucose reader. The estimations given with 10 minutes frequency by the Abbott Freestyle Navigator are plotted on the dashed line. The plus points correspond to blood glucose measurements made by HemoCue meter (references).

elements from the ordered set $\mathbf{z} ; \mathbf{z}_{T}=\mathbf{z} \backslash \mathbf{z}_{P}$. Then the kernel

$$
K^{0}(x, u)=(x u)^{0.89}+0.5 e^{-0.0003(x-u)^{2}}
$$

was chosen as an approximate minimizer of the functional (12), (15) with $\mu=0.5$, and $\lambda=\lambda(K)$ is given by the quasi-balancing principle (6)-(9) with $\lambda_{0}=10^{-4}, q=1.01$. The approximate minimizer was found by minimizing the functional $Q_{\mu}(K, \lambda(K), \mathbf{z})$ of the form (12), (15) on the set (13). The minimization has been performed by full search over the grid of parameters $\alpha_{i}=10^{-4} i, \beta_{j}=10^{-4} j, \gamma_{l}=$ $10^{-4} l, \quad i, j, l=1,2, \ldots, 3 \cdot 10^{4}$. Of course, the application of the full search method in finding the minimum of (12), (15) is computationally intensive, but in the present context it can be performed off-line.

For all nine subjects this kernel $K^{0}$ was used to construct a regularized estimators (5) that, starting from a raw electric signal $x \in[1,1024]$, return a blood glucose concentration $y=f_{K^{0}, \mathbf{z}}^{\lambda\left(K^{0}\right)}(x)$, where $\mathbf{z}=\left\{\left(x_{i}, y_{i}\right)\right\}_{i=1}^{30}$ are subject's data collected during the first day, and $\lambda\left(K^{0}\right)$ was chosen from (6) in accordance with the quasi-balancing principle (9) for the kernel $K^{0}$.

\section{A. Assessment of the blood glucose reading using Clarke $E G A$}

To quantify the clinical accuracy of constructed regularized blood glucose estimators we use the original Clarke EGA, which is accepted as one of the "gold standards" for determining the accuracy of blood glucose meters [20]. Note that another accuracy assessment metric that was originally developed to quantify the clinical accuracy of CGM systems is the continuous glucose-error grid analysis (CG-EGA) [21]. But to make use of the CG-EGA, the real blood glucose measurements must be sampled every 10-15 minutes or even more frequently (see [21], [22]). Keeping in mind that we operate only with restricted amount of real blood glucose measurements, in the present context we cannot use the CGEGA as an assessment metric. 


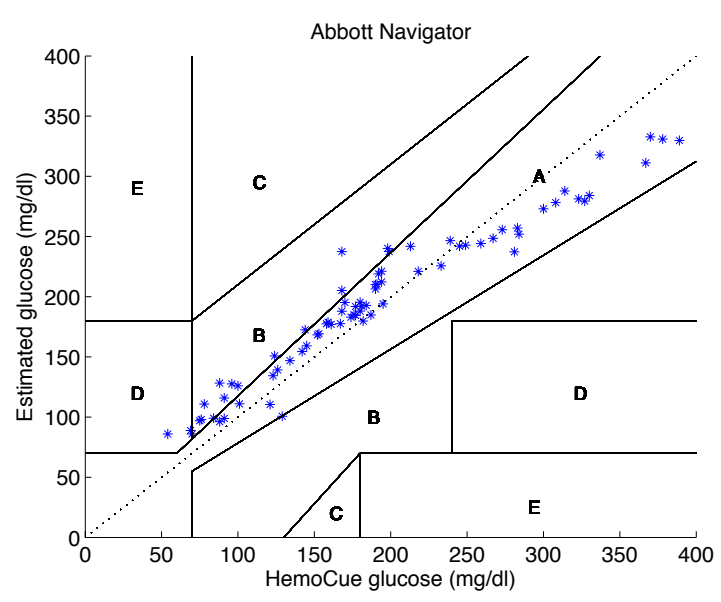

Fig. 2. Clarke EGA for Abbott Freestyle Navigator

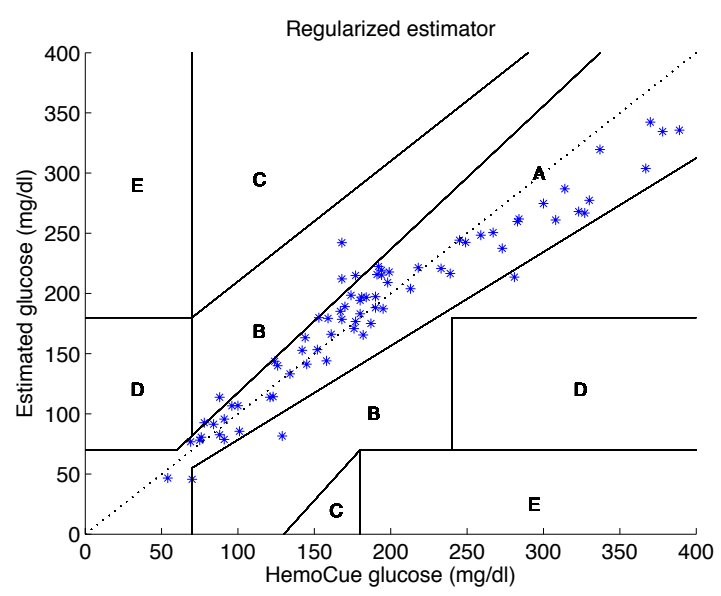

Fig. 3. Clarke EGA for Regularized estimator

In accordance with EGA methodology, for each of the nine subjects the available blood glucose values obtained by the HemoCue meter have been compared with the estimates of the blood glucose $y=f_{K^{0}, \mathbf{z}}^{\lambda\left(K^{0}\right)}(x)$. Here $x$ is a subcutaneous electric current value at the moment when corresponding HemoCue measurement was executed. Since HemoCue measurements made during the first day have been used for constructing regularized estimators only the data from another 2 days (at least $60 \mathrm{HemoCue}$ measurements) have been used as references in the Clarke's analysis.

In this analysis each pair (reference value, estimated value) identifies a point in the Cartesian plane, where the positive quadrant is subdivided into five zones, A to E, of varying degrees of accuracy and inaccuracy of glucose estimations (see Fig. 2, for example). Points in zones A and B represent accurate or acceptable glucose estimations. Points in zone $\mathrm{C}$ may prompt unnecessary corrections that could lead to a poor outcome. Points in zones D and E represents a dangerous failure to detect and treat. In short, the more points that appear in zones A and B, the more accurate the estimator is in terms of clinical utility.

\begin{tabular}{|c|c|c|c|c|c|}
\hline Subject & $A$ & $B$ & $C$ & $D$ & $E$ \\
\hline CHU102 & 86.42 & 13.58 & - & - & - \\
CHU105 & 87.5 & 11.25 & - & 1.25 & - \\
CHU 111 & 88.46 & 10.26 & - & 1.28 & - \\
CHU115 & 92.21 & 7.79 & - & - & - \\
CHU116 & 92.11 & 7.89 & - & - & - \\
CHU119 & 89.87 & 10.13 & - & - & - \\
CHU 128 & 87.76 & 12.24 & - & - & - \\
UNIPD202 & 81.08 & 17.57 & - & 1.35 & - \\
UNIPD203 & 93.33 & 6.67 & - & - & - \\
\hline Average & 88.75 & 10.82 & - & 0.43 & - \\
\hline
\end{tabular}

TABLE I

PERCENTAGE OF POINTS IN EGA-ZONES FOR ESTIMATORS BASED ON THE KERNEL (16)

A representative Clarke error grid (subject UNIPD203) for the proposed regularized blood glucose estimator is shown in Fig. 3. For comparison, in Fig. 2 the results of the EGA for blood glucose estimations determined from the internal readings of the Abbott Freestyle Navigator, calibrated according to the manufacturer's instruction, are presented for the same subject and reference values. Comparison shows that regularized estimator is more accurate, especially in case of low blood glucose. It can be also seen from the Fig. 1.

The results of the EGA for all subjects are summarized in Table I (regularized estimator) and Table II (Abbott Freestyle Navigator). From the D-columns in these tables one may conclude that the regularized estimator is, on average, at least 4 times more accurate than the considered commercial system.

To make the presented results even more transparent, let us closely look at the blood glucose reading in case of low blood glucose for which errors may have important clinical implications for patient health and safety. From Table III one can see that the Abbott Freestyle Navigator failed in detection of $66.7 \%$ of hypoglycemic events, whereas regularized estimator detected $75 \%$ of such events accurately.

From the comparison of these tables it is clear that in the considered clinical trial the proposed blood glucose estimators outperform the commercially available CGM systems that use the same input information. Moreover, in Table IV we also present the results of the EGA for the blood glucose estimator constructed with the use of the kernel (16) and the training set of only one subject CHU102. The exhibited performance was demonstrated in a 3-day test without any calibration to other subjects. Nevertheless, the clinical accuracy of the estimator is still acceptable, compared to required $94 \%$ in A+B-zone and $60 \%$ in Azone [23]. This result demonstrates the potential portability of a regularized estimator from patient to patient with no calibration.

In Table II one may observe a poor performance of the commercial CGM system in the case of subject CHU128, which is the only one type 2 diabetic patient in the considered group. At the same time, as it can be seen from Table I, the regularized estimator performs well for this patient that may be seen as a robustness to a diabetes type. 


\begin{tabular}{|c|c|c|c|c|c|}
\hline Subject & $A$ & $B$ & $C$ & $D$ & $E$ \\
\hline CHU102 & 93.83 & 6.17 & - & - & - \\
CHU105 & 92.5 & 5 & - & 2.5 & - \\
CHU111 & 85.9 & 12.82 & - & 1.28 & - \\
CHU115 & 94.81 & 5.19 & - & - & - \\
CHU116 & 86.84 & 10.53 & - & 2.63 & - \\
CHU119 & 83.54 & 16.46 & - & - & - \\
CHU128 & 48.98 & 44.9 & - & 6.12 & - \\
UNIPD202 & 89.19 & 8.11 & - & 2.7 & - \\
UNIPD203 & 76 & 21.33 & - & 2.67 & - \\
\hline Average & 83.51 & 14.5 & - & 1.99 & - \\
\hline
\end{tabular}

TABLE II

Percentage of points in EGA-Zones for AbBott Freestyle NAVIGATOR

\begin{tabular}{|c|c|c|}
\hline & Accurate & Erroneous \\
\hline $\begin{array}{c}\text { Abbott Freestyle } \\
\text { Navigator } \\
\begin{array}{c}\text { Regularized } \\
\text { estimator }\end{array}\end{array}$ & 4 & 8 \\
\hline
\end{tabular}

TABLE III

QUANTIFICATION OF BLOOD GLUCOSE READINGS IN HYPO ZONE FOR ALL SUBJECTS IN TOTAL (12 HYPOGLYCEMIC EVENTS)

\section{CONCLUSION}

These results allow a conclusion that on average the proposed approach for reading blood glucose levels from subcutaneous electric current is more accurate than estimations given by the Abbott Freestyle Navigator on the basis of the standard calibration procedure. The proposed approach can be seen as an answer to the request [3] for “more sophisticated calibration procedure." We would like to stress that no recalibrations of regularized glucose estimators have been made during the assessment period. At the same time, recalibrations of the Abbott Freestyle Navigator should sometimes be made several times per day. Moreover, the proposed algorithm can provide the estimated glucose at any requested time unlike the existing CGM sensors which have

\begin{tabular}{|c|c|c|c|c|c|}
\hline Subject & $A$ & $B$ & $C$ & $D$ & $E$ \\
\hline CHU102 & 84.55 & 15.45 & - & - & - \\
CHU105 & 52.72 & 45.45 & - & 1.82 & - \\
CHU111 & 77.78 & 22.22 & - & - & - \\
CHU115 & 39.25 & 60.75 & - & - & - \\
CHU116 & 60.29 & 38.24 & - & 1.47 & - \\
CHU 119 & 85.32 & 14.68 & - & - & - \\
CHU128 & 45.57 & 54.43 & - & - & - \\
UN IPD202 & 86.54 & 13.46 & - & - & - \\
UNIPD203 & 37.38 & 57.94 & 1.87 & 2.81 & - \\
\hline Average & 63.27 & 35.85 & 0.21 & 0.68 & - \\
\hline
\end{tabular}

TABLE IV

Percentage of PoINTS IN EGA-ZONES For the ESTIMATOR CONSTRUCTED WITH THE USE OF THE KERNEL (16) AND THE TRAINING SET OF ONLY ONE SUBJECT CHU 102. THE EXHIBITED PERFORMANCE WAS DEMONSTRATED IN A 3-DAY TEST WITHOUT ANY CALIBRATION TO OTHER SUBJECTS fixed sampling frequency.

\section{ACKNOWLEDGMENT}

This study has been pursued in the European FP7-project "DIAdvisor" [19].

\section{REFERENCES}

[1] The Diabetes Control and Complications Trial Research Group, The effect of intensive treatment of diabetes on the development and progression of long-term complications in insulin-dependent diabetes mellitus, The New England Jour. of Medicine, Vol. 329 (14), 1993, pp 977-986.

[2] H. Zisser, L. Robinson, W. Bevier, E. Dassau, C. Ellingsen, F.J. Doyle, and L. Jovanovic, Bolus Calculator: A Review of Four Smart Insulin Pumps, Diabetes Technology \& Therapeutics December 2008, Vol. 10 (6): pp 441-444.

[3] A. Facchinetti, G. Sparacino, and C. Cobelli, Reconstruction of glucose in plasma from interstitial fluid continuous glucose monitoring data: Role of sensor calibration, J. of Diabetes Sci. and Tech., Vol. 1 (5), 2007, pp 617-623.

[4] J. Reifman, S. Rajaraman, A. Gribok, and W. K. Ward, Predictive monitoring for improved management of glucose levels, J. of Diabetes Sci. and Tech., Vol. 1 (4), 2007, pp 478-486.

[5] G. Wahba, Splines Models for Observational Data, Series in Applied Mathematics, Vol. 59, SIAM, Philadelphia, PA, 1990.

[6] A.N. Tikhonov and V.B. Glasko, Use of the regularization methods in non-linear problems, USSR Comput. Math. Math. Phys., Vol. 5, 1965, pp 93-107.

[7] H. Engl, M. Hanke, and A. Neubauer, Regularization of Inverse Problems, Kluwer Academic Publishers, 1996.

[8] E. De Vito, S.V. Pereverzev and L. Rosasco, Adaptive Kernel Methods Using the Balancing Principle, Found. Comput. Math., Vol. 10, 2010, pp 455-479.

[9] G.R.G. Lanckriet, N. Christianini, L.E. Ghaoui, P. Bartlett and M.I. Jordan, Learning the kernel matrix with semidefinite programming, $J$. of Machine Learning Research, Vol. 5, 2004, pp 27-72.

[10] C.A. Micchelli and M. Pontil, Learning the kernel function via regularization, J. of Machine Learning Research, Vol. 6, 2005, pp 1099-1125.

[11] V. Naumova, S.V. Pereverzyev, and S. Sampath, Extrapolation in variable RKHSs with application to the blood glucose reading, Inverse Problems, Vol. 27, 075010, 2011, 13 pp.

[12] J. Mercer, Functions of positive and negative type and their connection with the theory of integral equations, Philos. Trans. R. Soc. Lond. Ser. A Math. Phys. Eng. Sci., Vol. 209, 1901, pp 415-446.

[13] T. Evgeniou, M. Pontil, and T. Poggio, Regularization Networks and Support Vector Machines, Advances in Computational Mathematics, Vol. 13, 2000, pp 1-50.

[14] N. Aronszajn, Theory of reproducing kernels, Trans. Amer. Math. Soc., Vol. 68, 1950, pp 337-404.

[15] F. Bauer, S.V. Pereverzev, and L. Rosasco, On regularization algorithms in learning theory, J. of Complexity, Vol. 23, 2007, pp 52-72.

[16] S. Kindermann and A. Neubauer, On the convergence of the quasioptimality criterion for (iterated) Tikhonov regularization, Inverse Problems and Imaging, Vol. 2 (2) , 2008, pp 291-299.

[17] O. Bousquet and D.J.L. Herrmann, On the complexity of learning the kernel matrix, Advances in Neural Information Processing Systems, Vol. 15, 2003.

[18] B.P. Kovatchev, M. Straume, D.J. Cox, and L.S. Farhi, Risk analysis of blood glucose data: a quantitative approach to optimizing the control of insulin dependent diabetes, J Theor Med., Vol. 3, 2000, pp 1-10.

[19] http://www.diadvisor.eu/

[20] W.L. Clarke, D.J. Cox, L.A. Gonder-Frederick, W. Carter, and S.L. Pohl, Evaluating clinical accuracy of systems for self-monitoring of blood glucose, Diabetes Care, Vol. 10 (5), 1987, pp 622-628.

[21] B.P. Kovatchev, L.A. Gonder-Frederick, D.J. Cox and W.L. Clarke, Evaluating the accuracy of continuous glucose-monitoring sensors: Continuous glucose-error grid analysis illustrated by TheraSense Freestyle Navigator, Diabetes Care, Vol. 27 (8), 2004, pp 1922-1928.

[22] W.L. Clarke, The original Clarke error grid analysis (EGA), Diabetes Technol Ther., Vol. 7(5), 2005, pp 776-779.

[23] http://www.childrenwithdiabetes.com/continuous.htm/ 